\title{
Genotypic Tests for Determining Coreceptor Usage of HIV-1
}

\author{
Daniel R. Kuritzkes \\ Section of Retroviral Therapeutics, Brigham and Women's Hospital and Harvard Medical School, Boston, Massachusetts
}

(See the article by Luke C. Swenson et al, on pages 237-245.)

Human immunodeficiency virus type 1 (HIV-1) relies on 2 receptors to infect target cells-CD4, the primary receptor, and either $\mathrm{C}-\mathrm{C}$ chemokine receptor 5 (CCR5) or C-X-C chemokine receptor 4 (CXCR4), which serve as coreceptors. Most infections involve transmission of CCR5-using (R5) virus, but over the course of infection CXCR4-using virus emerges in about half of patients. The CCR5 antagonist maraviroc inhibits entry of R5 virus, but it has little or no activity against CXCR4-using virus $[1,2]$. For this reason, coreceptor usage (often referred to as "tropism") of a patient's virus must be assessed in order to determine whether maraviroc is an appropriate drug for inclusion in an antiretroviral regimen. Clinical trials of maraviroc and other chemokine receptor antagonists have relied on a phenotypic assay of coreceptor usage (Trofile, Monogram BioSciences) that utilizes pseudotyped viruses constructed from envelope sequences amplified from plasma virus by reverse transcriptase-coupled PCR [3].

Received 5 October 2010; accepted 6 October 2010.

Potential conflicts of interest: The author is a consultant to Pfizer, Roche, Tobira, and ViiV.

Reprints or correspondence: Dr Daniel R. Kuritzkes, Section of Retroviral Therapeutics, Brigham and Women's Hospital, 65 Lansdsowne St, Rm 449, Cambridge, MA 02139 (dkuritzkes@partners.org).

The Journal of Infectious Diseases 2011;203:146-148 (C) The Author 2011. Published by Oxford University Press on behalf of the Infectious Diseases Society of America. All rights reserved. For Permissions, please e-mail: journals. permissions@oup.com

1537-6613/2011/2032-0001\$15.00

DOI: 10.1093/infdis/jiq034
Although phenotypic tests of coreceptor usage are generally considered the gold standard, the expense and long turnaround time (4-6 weeks) of these assays have spurred the development of genotypic methods as an alternative. Genotypic determination of HIV-1 coreceptor usage is based on sequencing the V3-coding region of HIV-1 env, the principal determinant of coreceptor usage. A variety of algorithms and bioinformatics programs can be used to predict coreceptor usage from the V3 sequence. When compared with the phenotypic assay, genotypic methods show high specificity $(\sim 90 \%)$ but only modest sensitivity (50\%-70\%; reviewed in [4]). Factors that contribute to the limited sensitivity of genotypic assays include determinants of coreceptor usage that lie outside of V3 and the difficulty of detecting the presence of minority CXCR4-using species when performing population sequencing of env.

In the current issue of the Journal, Swenson et al [5] describe the application of deep sequencing to predict the tropism of HIV-1 in plasma samples from patients screening for enrollment into 1 of 3 clinical trials of maraviroc. Deep sequencing, sometimes referred to as massively parallel sequencing, can generate sequences from each of the thousands of HIV-1 genomes present in a plasma sample at a faster speed and to a greater depth than conventional cloning or single-genome sequencing [6]. Because deep sequencing can resolve the complex heterogeneity of V3 loop sequences present in clinical samples, this next-generation sequencing approach could provide much greater sensitivity for detecting CXCR4-using variants than population sequencing.

Coreceptor usage of plasma virus from samples obtained at screening for the pivotal phase 3 trials of maraviroc (Maraviroc versus Optimized Therapy in Viremic Antiretroviral Treatment-Experienced Patients [MOTIVATE] [1]) was determined using ultradeep sequencing of V3, and the results were compared with phenotypic testing performed by the original Trofile assay. Samples in which $\geqslant 2 \%$ of the V3 sequences predicted CXCR4 usage by the position sequence scoring matrix were considered non-R5. Virologic responses of subjects with R5 or non-R5 virus who received maraviroc in the MOTIVATE trials or in the companion A4001029 study were analyzed [2]. Only patients with R5 virus by Trofile were eligible for MOTIVATE, but a limited number of treatment-experienced subjects with DM or X4 virus at screening, or for whom no Trofile result was available, were enrolled into the A4001029 study.

Deep sequencing was performed on samples from 1827 screening samples from the 3 trials. Overall concordance between the deep sequencing and phenotypic approaches was $82 \%$. Of note, slightly more patients were considered to have DM or X4 virus by deep sequencing than by phenotypic assay (43\% vs $38 \%$, 
respectively). When the Trofile assay was taken as the gold standard, then deep sequencing had a sensitivity of $84 \%$ and a specificity of $81 \%$ for detecting presence of DM or X4 virus. Conversely, when deep sequencing was taken as the gold standard, the Trofile assay had a sensitivity of $75 \%$ and a specificity of $89 \%$. Deep sequencing appeared to be more sensitive, if somewhat less specific, in detecting DM or X4 virus as compared with standard (population-based) sequencing of V3: Standard genotyping had a sensitivity and specificity of $67 \%$ and 93\%, respectively, using Trofile as the gold standard, and $64 \%$ and $93 \%$, respectively, using deep sequencing as the gold standard.

Genotyping predicted the virologic response to maraviroc therapy as well as phenotyping: the median week 8 change in plasma HIV-1 RNA for patients categorized as having $\mathrm{R} 5$ virus by genotype was $-2.4 \log _{10}$ copies/mL, which was identical to the median week 8 change in virus load in patients classified as having R5 virus by Trofile $\left(-2.4 \log _{10}\right.$ copies $/ \mathrm{mL}$ ). By contrast, patients found to have CXCR4-using virus by genotype or Trofile had smaller median virus load declines in response to maraviroc $(-1.4$ and $-1.3 \log _{10}$ copies/mL, respectively). Only a small number of subjects $(n=6)$ had discordant results in which genotyping suggested the presence of R5 virus but Trofile suggested non-R5 virus; the median change in plasma HIV-1 RNA at week 8 in this group was -2.6 $\log _{10}$ copies/mL. Deep sequencing and phenotyping performed equally well at predicting the 48-week response to maraviroc: $49 \%$ classified as R5 by genotype and $46 \%$ classified as R5 by Trofile achieved a plasma virus load of $<50$ copies/mL at week 48 ; those classified as having CXCR4-using virus by genotype or Trofile had response rates of $26 \%$ and 23\%, respectively. Of note, there appeared to be an inverse gradient of virologic response to maraviroc based on the percentage of CXCR4-using virus detected in the screening sample.
Establishing an appropriate cutoff for detecting CXCR4-using virus is a challenge for both phenotypic and genotypic assays. The original Trofile assay reliably detected $\mathrm{X} 4$ variants present at $10 \%$ of the viral population, but only detected variants present at $5 \%$ of the population in $85 \%$ of assays [3]. The current enhanced Trofile assay is validated to detect X4 variants present at $.3 \%$ of the population with $100 \%$ sensitivity [7]. However, actual sensitivity in clinical samples may vary as a function of virus load and infectivity of the virus being assayed. In clinical trials, nearly half of maraviroc or vicriviroctreated subjects who ultimately experienced virologic failure with emergence of CXCR4-using virus were classified as R5 at screening by the enhanced Trofile assay $[7,8]$.

In the study by Swenson et al, deep sequencing was more sensitive than either population sequencing or phenotyping at detecting the presence of CXCR4-using virus [5]. The greater sensitivity of deep sequencing was particularly evident when non-R5 virus was present at $2 \%-20 \%$ of the population. A threshold of $2 \%$ was selected empirically, based on optimizing the week 8 responses to maraviroc of a random portion of the data set, and based on the reproducibility of PCR. As with the enhanced Trofile assay, this threshold may not be sufficiently sensitive-only $40 \%$ of patients in whom $\mathrm{DM}$ or $\mathrm{X} 4$ virus emerged while receiving maraviroc were classified as non-R5 by deep sequencing, using the 2\% cutoff; CXCR4-using virus emerged in $18 \%$ of patients with R5 virus by genotype at screening. Failure to detect minority CXCR4-using variants may pose the greatest risk in treatmentnaïve patients initiating a maraviroccontaining regimen, as selection of previously undetected DM or X4 variants may lead to virologic failure accompanied by resistance to other components of the regimen [8]. Despite these limitations, deep sequencing performed as well as the Trofile assay in predicting the outcome of maraviroc treatment.

Given these results, is it now appropriate to recommend genotypic testing to determine HIV-1 coreceptor usage when considering treatment with a CCR5 antagonist? Although the data reported by Swenson et al [5] go a long way toward establishing genotyping as a suitable alternative to phenotypic testing to predict coreceptor usage, the analysis falls short of a formal prospective validation. A significant limitation of the current analysis is that all the patients who received maraviroc were prescreened by the original Trofile assay. For this reason, it is not possible to estimate the true sensitivity and specificity of deep sequencing for predicting treatment response to maraviroc. The same limitation applies to the enhanced Trofile assay, which has only been validated retrospectively in studies that used the original Trofile assay to determine eligibility for CCR5 antagonist therapy. A second limitation is that deep sequencing is compared with the original Trofile assay, which is no longer available. Whether sequencing would compare as favorably to the enhanced sensitivity Trofile assay remains open to conjecture. Finally, it is important to note that not all genotypic assays offered by clinical laboratories replicate the meticulous approach taken by the authors of the current report. Given the limited number of samples available from a handful of adequately powered clinical trials of CCR5 antagonists, validating alternative genotypic tests of coreceptor usage remains a major challenge for the field.

\section{References}

1. Gulick RM, Lalezari J, Goodrich J, et al. Maraviroc for previously treated patients with R5 HIV-1 infection. N Engl J Med 2008; 359:1429-41.

2. Saag M, Goodrich J, Fatkenheuer G, et al. A double-blind, placebo-controlled trial of maraviroc in treatment-experienced patients infected with non-R5 HIV-1. J Infect Dis 2009; 199:1638-47.

3. Whitcomb JM, Huang W, Fransen S, et al. Development characterization of a novel 
single-cycle recombinant-virus assay to determine human immunodeficiency virus type 1 coreceptor tropism. Antimicrob Agents Chemother 2007; 51:566-75.

4. Lin NH, Kuritzkes DR. Tropism testing in the clinical management of HIV-1 infection. Curr Opin HIV AIDS 2009; 4:481-7.

5. Swenson LC, Mo T, Dong WWY, et al. Deep sequencing to infer HIV-1 co-receptor usage:
Application to three clinical trials of maraviroc in treatment-experienced patients. J Infect Dis 2010. doi:10.1093/jid/jiq030.

6. Tsibris AM, Korber B, Arnaout R, et al. Quantitative deep sequencing reveals $\mathrm{dy}$ namic HIV-1 escape and large population shifts during CCR5 antagonist therapy in vivo. LoS One 2009; 4:e5683.

7. Su Z, Gulick RM, Krambrink A, et al. Response to vicriviroc in treatment-experienced subjects, as determined by an enhancedsensitivity coreceptor tropism assay: reanalysis of AIDS clinical trials group A5211. J Infect Dis 2009; 200:1724-8.

8. Cooper DA, Heera J, Goodrich J, et al. Maraviroc versus efavirenz, both in combination with zidovudine-lamivudine, for the treatment of antiretroviral-naive subjects with CCR5-tropic HIV-1 infection. J Infect Dis 2010; 201:803-13. 